\title{
Elderly kidney transplant recipients have favorable outcomes but increased infection-related mortality
}

Gayoung Lee, Jeonghoon Lim, Jihye Kim, Soojee Jeon, Heewon Noh, Jiyoung Choi, Janghee Cho, Sunhee Park, Yonglim

Kim, Chanduck Kim

Department of Internal Medicine, Kyungpook National University Hospital, Daegu, Korea

Background: Elderly patients with end-stage kidney disease have been increasing. However, the outcomes of kidney transplantation (KT) in elderly patients is unclear, particularly in Asian kidney transplant recipients (KTRs). We evaluated the clinical outcomes of elderly KTRs and analyzed the impact of elderly donors.

Methods: This is a retrospective cohort study including patients who underwent KT between January 2000 and December 2019 at the Kyungpook National University Hospital. KTRs were divided into four groups, according to recipient and donor age ( $\geq 60$ years or not) combination: elderly recipients: old-to-old $(n=46)$ and young-to-old $(n=83)$; young recipients: old-to-young $(n=98)$ and young-to-young $(n=796)$. We compared the risk of mortality, death-censored graft failure, and biopsy-proven acute rejection (BPAR) using Cox regression analysis.

Results: The incidences of delayed graft function, graft failure, and BPAR were not different among groups (all P>0.05). Annual mean tacrolimus trough level was not lower in elderly recipients compared to young recipients during 10 -year follow-up. The mortality was significantly higher in elderly recipients compared to young recipients $(P=0.001)$; in particular, infection-related mortality was higher in elderly recipients $(P<0.001)$. In the multivariate Cox regression analysis, old-to-old and young-to-old groups had increased mortality risk than young-to-young group (adjusted hazard ratio [aHR], 3.16; 95\% confidence interval [Cl], 1.26-7.97; $\mathrm{P}=0.014 ;$ aHR, 3.14; $95 \% \mathrm{Cl}, 1.57-6.30 ; \mathrm{P}=0.001$, respectively). However, graft failure and BPAR risk were not increased in elderly recipients than young-to-young group (both $\mathrm{P}>0.05$ ).

Conclusions: In the elderly recipients, graft survival and BPAR-free survival were not inferior to those of young recipients, but mortality, especially risk of infection-related death was increased. Low immunosuppression intensity might help decrease mortality in elderly recipients.

Corresponding author: Gayoung Lee

E-mail: aiyaiyai@naver.com

(c) The Korean Society for Transplantation

This is an Open Access article distributed under the terms of the Creative Commons Attribution Non-Commercial License (http://creativecommons.org/licenses/by-nc/4.0/) which permits unrestricted non-commercial use, distribution, and reproduction in any medium, provided the original work is properly cited. 